\title{
In-situ TEM Investigation on Thermal Stability and Oxygen Release Behavior of Charged and Discharged $\mathrm{LiCoO}_{2}$
}

\author{
Soroosh Sharifi-As1 ${ }^{1}$, Yifei Yuan ${ }^{2}$, Hasti Asayesh-Ardakan ${ }^{2}$, Anmin Nie $^{1}$, Robert F Klie ${ }^{3}$, Reza \\ Shahbazian-Yassar ${ }^{1}$ \\ 1. Mechanical and industrial Engineering Department, University of Illinois at Chicago, Chicago, IL, \\ United States. \\ 2. Department of Mechanical Engineering, Michigan Technological University, Houghton, MI, United \\ States. \\ 3. Department of Physics, University of Illinois at Chicago, Chicago, IL, United States.
}

Since the first discovery of Li ion batteries (LIBs), improving electrochemical properties such as power density and energy density of batteries was the main concern of researchers. Yet not much research has been focused on the safety issues and thermal stability of these systems.

$\mathrm{LiCoO} 2$ is one of the most widely used cathode materials in LIBs. High energy density and light weight cell has made it a good candidate for first generation electric vehicles. However, due to the poor thermal stability, its application has been limited to the consumer electronics now. It is well known that Charged LCO when exposed to higher temperatures, as in a case of a mechanical impact or overcharge, releases a portion of its oxygen. Released oxygen can react with electrolyte exothermically and trigger a hazardous thermal runaway. In order to hinder this harmful process we need to gain more in-depth knowledge about underlying mechanisms of this harmful reaction and phase/structural transitions $[1,2]$.

In-Situ TEM is known to be a powerful method for studying materials in dynamic conditions such as high temperatures. In this study we utilized transmission electron microscopy to study $\mathrm{Li}_{1-\mathrm{x}} \mathrm{CoO}_{2}$ $(0.5<x<1)$ in-situ in different temperatures. Utilizing powerful techniques such as electron energy loss spectroscopy, selected area diffraction and high resolution STEM imaging helps us in this research to study thermal stability and oxygen release behavior of LCO in different states of charge exposed to high temperatures.

As we expected, discharged LCO $(\mathrm{x}=1)$ is stable at relatively high temperatures and no phase transition and structural deterioration was detected up to $450^{\circ} \mathrm{C}$. On the other hand charged LCO shows a completely different behavior. Figure 1 shows SAD patterns obtained from charged LCO at different temperatures. It can be seen that in temperatures as high as $200^{\circ} \mathrm{C}$, spinel pattern and $\mathrm{Li}$ vacancy super lattice is added to Pattern of original layered structure of charged LCO particles. Interestingly, the newly formed spinel structure then disappears from SAD patterns at temperatures higher than $400^{\circ} \mathrm{C}$. Additionally, in morphological TEM images, regions with inhomogeneous contrast appears at high temperatures mostly close to edges. To understand more details about atomic arrangement after a heating cycle high resolution STEM imaging was performed on the fully charged LCO which was heated to $300^{\circ} \mathrm{C}$. It was seen that atomic arrangement close to the edge $(0-15 \mathrm{~nm})$ of the particles is totally different from the previous atomic resolution images. Obtained images from core part of particles indicates that original layered structure is still unchanged. This results suggest that, a layer of transformed spinel structure forms around LCO particles at elevated temperatures $\left(\sim 200^{\circ} \mathrm{C}\right)$. This newly formed structure is unstable when going to higher temperatures $\left(\sim 400^{\circ} \mathrm{C}\right)$, and oxygen release happens mostly from this part of sample. therefore spinel structure collapses and pattern disappears from SADP 
after $400^{\circ} \mathrm{C}$. Areas with lower contrast can be result of oxygen release and porosity formation and high contrast areas can be due to formation of cobalt oxides phases with higher average atomic number such as $\mathrm{CoO}$.

To have a better understanding of the structural changes and to be able to monitor oxygen release behavior, electron energy loss spectroscopy (EELS) can be advantageous. While in pristine sample there is no changes in terms of electronic structure, spectra from charged LCO particles suggests apparent changes in valence state of cobalt atoms in different temperatures (figure1). By increasing the temperature, oxygen K-edge pre-edge peak shifts to higher energies which indicates reduction of cobalt atoms around the oxygen sites. Apparent changes also occur in the $\mathrm{Co}_{3,2}$ edges. To be able to precisely determine the onset temperature of oxygen release in specimens, calibration has been done with standard sample with different valence of cobalt. Calibration results suggests that energy difference between L3 and L2 peaks and oxygen pre-edge and main peak are the best method to distinguish different state of charge of cobalt atoms. Finally an EELS line scan from edge to core part of a charged LCO at $450^{\circ} \mathrm{C}$ confirms that oxygen release is mostly happening and cobalt valence is changing to $2+$ close to the edge, however spectra from core part indicates valence state of Co is pretty consistent with the one from room temperature and there is not obvious changes in cobalt valence state in this region [3].

\section{References:}

[1] Y Furushima et al, Journal of Power Sources 196 (2011), p. 2260.

[2] J Geder et al, Journal of Power Sources 257 (2014), P. 286.

[3] The authors acknowledge funding from National Science Foundation -NSF CMMI-1619743.
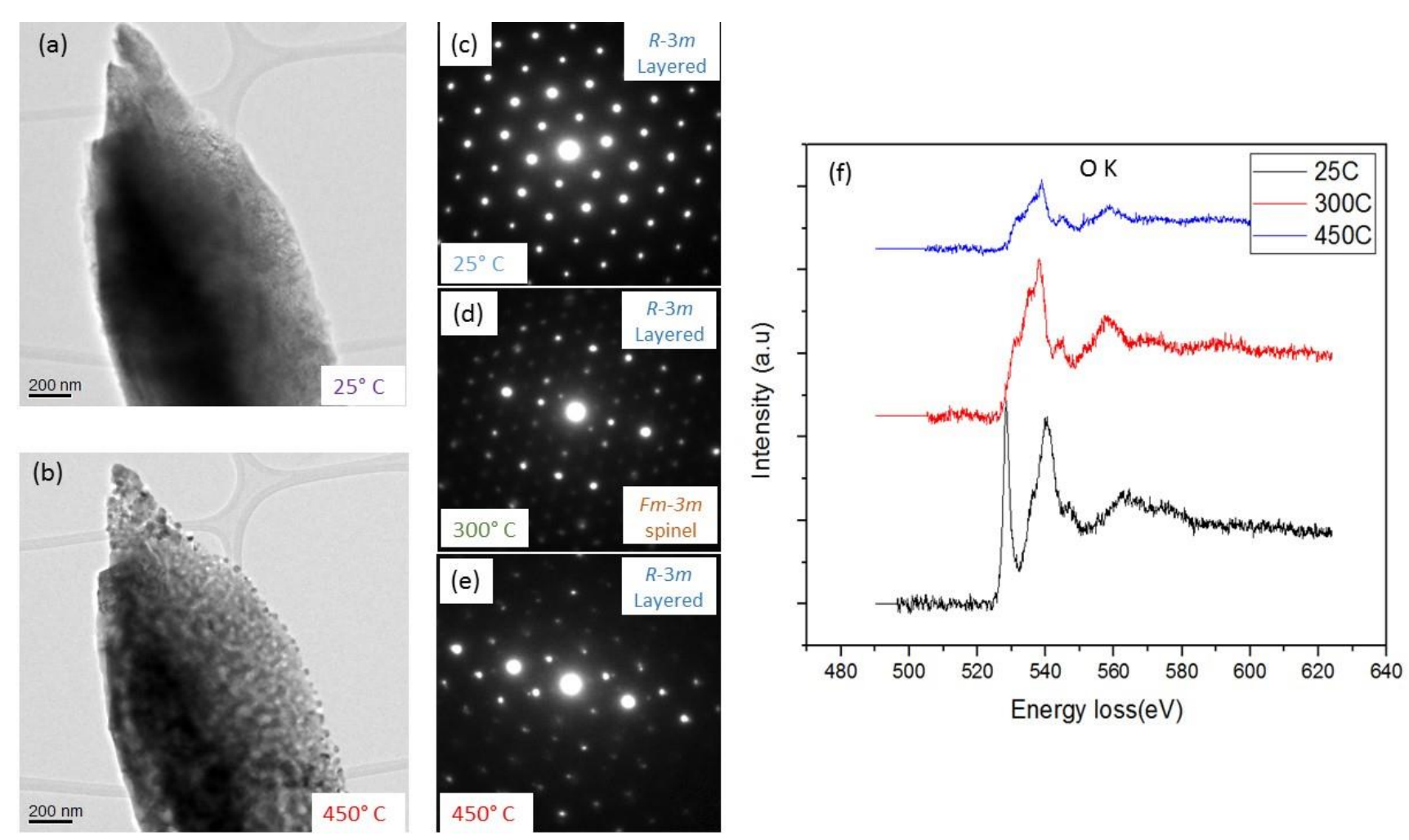

Figure 1. (a, b) Morphological TEM images from a charged LCO particle at $25^{\circ}$ and $450^{\circ} \mathrm{C}$. (c, d, e, f,) SAD patterns from a charged LCO particle at shown temperatures. (f) EEL spectra from the oxygen K edge in different temperatures. 